University of Wollongong

Research Online

Faculty of Engineering - Papers (Archive)

Faculty of Engineering and Information

Sciences

$1-1-2009$

\title{
Superconductivity of various borides: The role of stretched c-parameter
}

\author{
Monika Mudgel \\ National Physical Laboratory India \\ V P. Awana \\ National Physical Laboratory, New Delhi \\ H Kishan \\ National Physical Laboratory, New Delhi, India \\ I Felner \\ Hebrew University of Jerusalem \\ Gustavo A. Alvarez \\ University of Wollongong, gustavo@uow.edu.au
}

See next page for additional authors

Follow this and additional works at: https://ro.uow.edu.au/engpapers

Part of the Engineering Commons

https://ro.uow.edu.au/engpapers/3258

\section{Recommended Citation}

Mudgel, Monika; Awana, V P.; Kishan, H; Felner, l; Alvarez, Gustavo A.; and Bhalla, G L.: Superconductivity of various borides: The role of stretched c-parameter 2009, 07E313-1-07E313-3.

https://ro.uow.edu.au/engpapers/3258

Research Online is the open access institutional repository for the University of Wollongong. For further information contact the UOW Library: research-pubs@uow.edu.au 
Authors

Monika Mudgel, V P. Awana, H Kishan, I Felner, Gustavo A. Alvarez, and G L. Bhalla 


\section{AIP $\mid$ Appilied Physics}

\section{Superconductivity of various borides: The role of stretched c-parameter}

Monika Mudgel, V. P. S. Awana, H. Kishan, I. Felner, Dr. G. A. Alvarez et al.

Citation: J. Appl. Phys. 105, $07 E 313$ (2009); doi: 10.1063/1.3072719

View online: http://dx.doi.org/10.1063/1.3072719

View Table of Contents: http://jap.aip.org/resource/1/JAPIAU/v105/i7

Published by the American Institute of Physics.

\section{Related Articles}

Terahertz nonlinear superconducting metamaterials Appl. Phys. Lett. 102, 081121 (2013)

Penetration depth of MgB2 measured using Josephson junctions and SQUIDs Appl. Phys. Lett. 102, 072603 (2013)

Effect of excess $\mathrm{Mg}$ and nano-additives on the superconducting properties of weakly connected bulk MgB2 J. Appl. Phys. 113, 063908 (2013)

Current crowding effects in superconducting corner-shaped Al microstrips Appl. Phys. Lett. 102, 052603 (2013)

A three-dimensional strain model for the superconducting properties of strained International Thermonuclear Experimental Reactor Nb3Sn strands

J. Appl. Phys. 112, 113909 (2012)

\section{Additional information on J. Appl. Phys.}

Journal Homepage: http://jap.aip.org/

Journal Information: http://jap.aip.org/about/about_the_journal

Top downloads: http://jap.aip.org/features/most_downloaded

Information for Authors: http://jap.aip.org/authors

\section{ADVERTISEMENT}

\section{AIPAdvainces}

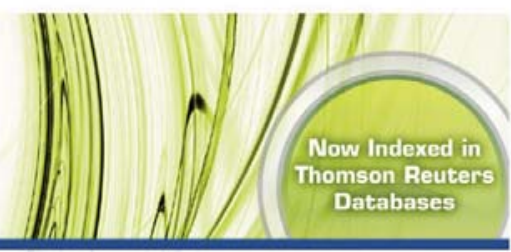

\section{Explore AIP's open access journal: •Rapid publication \\ - Article-level metrics \\ Post-publication rating and commenting}




\title{
Superconductivity of various borides: The role of stretched $c$-parameter
}

\author{
Monika Mudgel, ${ }^{1}$ V. P. S. Awana, ${ }^{1, a)}$ H. Kishan, ${ }^{1}$ I. Felner, ${ }^{2}$ Dr. G. A. Alvarez, ${ }^{3}$ and \\ G. L. Bhalla \\ ${ }^{1}$ National Physical Laboratory, Dr. K. S. Krishnan Marg, New Delhi-110012, India \\ ${ }^{2}$ Racah Institute of Physics, Hebrew University of Jerusalem, Jerusalem-91904, Israel \\ ${ }^{3}$ Institute for Superconducting and Electronic Materials, University of Wollongong, Australia \\ ${ }^{4}$ Department of Physics and Astrophysics, University of Delhi, Delhi-110007, India
}

(Presented 13 November 2008; received 16 September 2008; accepted 24 November 2008; published online 6 March 2009)

\begin{abstract}
The superconductivity of $\mathrm{MgB}_{2}, \mathrm{AlB}_{2}, \mathrm{NbB}_{2+x}$, and $\mathrm{TaB}_{2+x}$ is intercompared. The stretched $c$-lattice parameter $(c=3.52 \AA)$ of $\mathrm{MgB}_{2}$ in comparison to $\mathrm{NbB}_{2.4}(c=3.32 \AA)$ and $\mathrm{AlB}_{2}(c=3.25 \AA)$ decides empirically the population of their $\pi$ and $\sigma$ bands and as a result their transition temperature $T_{\mathrm{c}}$ values, respectively, at 39 and $9.5 \mathrm{~K}$ for the first two and no superconductivity for the later. The nonstoichiometry induces an increase in $c$ parameter with Boron excess both in $\mathrm{NbB}_{2+x}$ and $\mathrm{TaB}_{2+x}$. Magnetization $(M-T)$ and resistivity measurements $(\rho-T)$ in case of niobium boride samples show the absence of superconductivity in stoichiometric $\mathrm{NbB}_{2}$ sample $(c=3.26 \AA)$ while a clear diamagnetic signal and a $\rho=0$ transition for boron excess $\mathrm{NbB}_{2+x}$ samples. On the other hand, superconductivity is not achieved in $\mathrm{TaB}_{2+x}$ case. The probable reason behind is the comparatively lesser or insufficient stretching of $c$ parameter. () 2009 American Institute of Physics.
\end{abstract}

[DOI: 10.1063/1.3072719]

\section{INTRODUCTION}

The superconductivity in the family of $\mathrm{AlB}_{2}$ type diborides like $\mathrm{TaB}_{2}, \mathrm{NbB}_{2}$, and $\mathrm{ZrB}_{2}$ was boost up with the discovery of $\mathrm{MgB}_{2}$ superconductor. ${ }^{1}$ But in the comparison of $\mathrm{MgB}_{2}$, very few reports exist on other diborides; even the existence of superconductivity is suspected in some of the diborides. For example, $\mathrm{ZrB}_{2}$ is reported to have a $T_{\mathrm{c}}$ of 5.5 $\mathrm{K}$ by Gasprov et al., ${ }^{2}$ whereas Leyarovska and Leyarovski ${ }^{3}$ report no transition. Similarly, Gasprov et al. and others ${ }^{2-5}$ have reported no observation of superconductivity in $\mathrm{TaB}_{2}$, while Kackzorowski et al. ${ }^{6}$ report a transition temperature of $9.5 \mathrm{~K}$. The results for $\mathrm{NbB}_{2}$ are even more diverse. Gasprov et al., ${ }^{2}$ and Kackzorowski et al. and others ${ }^{6,7}$ report no superconductivity, while many others ${ }^{3,8,9}$ report different values of transition temperature in the range of $0.62-9.2 \mathrm{~K}$.

Band structure calculations in $\mathrm{MgB}_{2}$ reveal that $T_{\mathrm{c}}$ increases with increase in $c$ parameter. ${ }^{10}$ Working on the same idea, $\mathrm{NbB}_{2+x}$ and $\mathrm{TaB}_{2+x}$ samples are checked for existence of superconductivity and the systematic comparison in both $\mathrm{NbB}_{2+x}$ and $\mathrm{TaB}_{2+x}$ is carried out. The thermoelectric power of stoichiometric samples of $\mathrm{MgB}_{2}, \mathrm{AlB}_{2}$, and $\mathrm{NbB}_{2}$ is also intercompared.

\section{EXPERIMENTAL}

The polycrystalline samples of $\mathrm{MgB}_{2}, \mathrm{AlB}_{2}, \mathrm{NbB}_{2+x}(x$ $=0.0$ to 0.8$)$, and $\mathrm{TaB}_{2+x}(x=0.0$ to 0.8$)$ were prepared by simple solid-state reaction route. See our Refs. 11 and 12 . X-ray diffraction patterns done on Rigaku-Miniflex-II and

\footnotetext{
a) Author to whom correspondence should be addressed. FAX: 0091-1145609310. Tel.: 0091-11-45609210. Electronic mail: awana@mail.nplindia.ernet.
}

Rietveld analysis was done by Fullprof program-2007. For details of other measurements, see Refs. 12 and 13.

\section{RESULTS AND DISCUSSION}

Figure 1(a) shows the $\mathrm{x}$-ray diffraction patterns for $\mathrm{MgB}_{2}$ and $\mathrm{AlB}_{2}$, while Fig. 1(b) shows the same for $\mathrm{NbB}_{2}$, $\mathrm{NbB}_{2.4}, \mathrm{TaB}_{2}$, and $\mathrm{TaB}_{2.4}$ samples. In order to confirm the phase purity, Rietveld refinement is done for all the samples in the space group P6/mmm No. 191. There is hardly any difference between the experimentally observed and theoretically Rietveld determined $\mathrm{x}$-ray profiles except a small $\mathrm{MgO}$ peak in case of $\mathrm{MgB}_{2}$ shown by \#. We observe that the (002) peak shifts toward lower angle side with the boron excess in both $\mathrm{NbB}_{2}$ and $\mathrm{TaB}_{2}$ cases, which results in increase in $c$ parameter. The systematic variation in the parameters can be seen from Table I. There is a slight decrease in $a$ parameter with increasing boron content in both $\mathrm{NbB}_{2+x}$ and $\mathrm{TaB}_{2+x}$. In case of $\mathrm{NbB}_{2+x}, c$ parameter increases continuously up to $x$ $=0.4$ and then saturates further with negligible up and downs, but in $\mathrm{TaB}_{2+x}, c$ parameter increases considerably but only up to $x=0.2$ sample and saturates thereafter. The structural information is in well confirmation with the literature. ${ }^{6,14-16}$ Although the $a$ and $c$ values for $\mathrm{TaB}_{2+x}$ samples match quantitatively with the earlier reports ${ }^{5,16}$ but differ in respect to corresponding compositions. $\mathrm{MgB}_{2}$ is found to be a superconductor with $T_{\mathrm{c}}$ of about $39 \mathrm{~K}$ while $\mathrm{AlB}_{2}$ is a nonsuperconductor. ${ }^{11,13}$

Magnetization versus temperature $(M-T)$ plot including both zero field cooled and field cooled curves is shown in the main panel of Fig. 2(a) for $\mathrm{NbB}_{2.4}$ sample in the temperature range of 5-12 K. The $\mathrm{NbB}_{2.4}$ sample shows a clear diamagnetic signal at about $9.5 \mathrm{~K}$, implying that it is a superconductor. The lower inset in Fig. 2(a) shows magnetization versus temperature curves for $\mathrm{NbB}_{2}$ sample in the temperature 


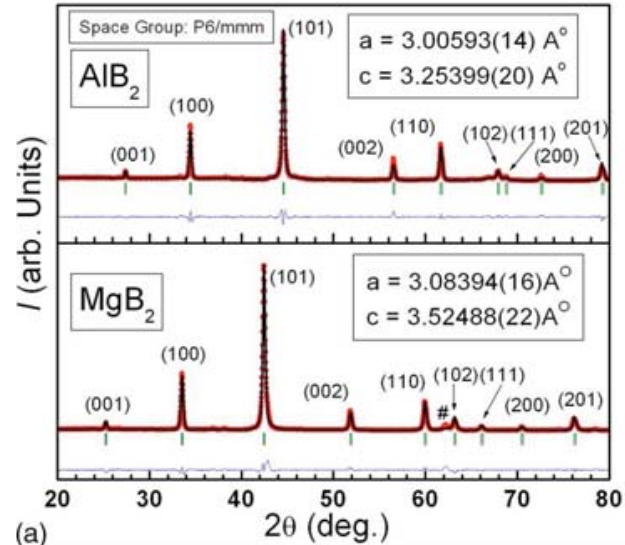

(a)

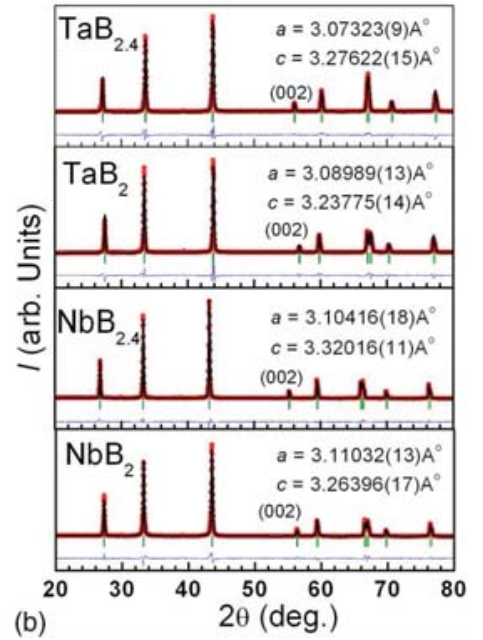

FIG. 1. (Color online) Rietveld refined plots for (a) $\mathrm{MgB}_{2}$ and $\mathrm{AlB}_{2}$ samples and (b) $\mathrm{NbB}_{2}, \mathrm{NbB}_{2.4}, \mathrm{TaB}_{2}$, and $\mathrm{TaB}_{2.4}$ samples. X-ray experimental diagram (dots), calculated pattern (continuous line), difference (lower continuous line), and calculated Bragg position (vertical lines in middle). range of 5-300 K. The sample exhibits no diamagnetic signal and hence possesses no bulk superconductivity. The magnetization measurement with varying field at a fixed temperature of $5 \mathrm{~K}$ is also done for the superconducting $\mathrm{NbB}_{2.4}$ sample and is shown in the upper inset. Thus, $\mathrm{NbB}_{2.4}$ is a type-II superconductor with the $H_{\mathrm{c} 1}$ and $H_{\mathrm{c} 2}$ values of 500 and $1600 \mathrm{Oe}$, respectively. In this way boron excess increases the $c$ parameter and induces superconductivity in niobium boride sample. All boron excess samples are found to possess superconductivity with different $T_{\mathrm{c}}$ values. $^{12}$

The main panel of Fig. 2(b) shows the $\rho$ - $T$ measurement for $\mathrm{NbB}_{2.4}$ sample, while the inset shows the same for $\mathrm{NbB}_{2}$ sample. The $\mathrm{NbB}_{2.4}$ sample shows a sharp transition with a $T_{\mathrm{c}}$ onset of $7.5 \mathrm{~K}$. On the other hand the stoichiometric $\mathrm{NbB}_{2}$ sample just shows metallic behavior from $300 \mathrm{~K}$ to about $T=80 \mathrm{~K}$. After that resistivity becomes almost constant and shows no superconducting transition down to $5 \mathrm{~K}$. Thus $\rho-T$ measurement is in confirmation with the $M-T$ measurement showing that only boron excess sample is superconducting, while the stochiometric $\mathrm{NbB}_{2}$ is a nonsuperconductor although $T_{\mathrm{c}}$ onset obtained from magnetization measurement for $\mathrm{NbB}_{2.4}$ is comparatively higher.

After inducing superconductivity in $\mathrm{NbB}_{2+x}$, the same is tried for $\mathrm{TaB}_{2+x}$ sample. The magnetization versus temperature measurements $(M-T)$ are shown in Fig. 3 for $\mathrm{TaB}_{2+x}$ samples in the temperature range of 5-20 K. The samples do not exhibit any diamagnetic signal confirming that there is no superconductivity below to $5 \mathrm{~K}$. The magnetic moment increases with the decrease in temperature for all the samples. The inset shows the magnetic behavior of $\mathrm{TaB}_{2.4}$ and $\mathrm{TaB}_{2.6}$ samples with varying field at a fixed temperature of $5 \mathrm{~K}$. The magnetic moment increases with the applied field and then saturates at a field of about $4 \mathrm{kOe}$ and a hysteresis is obtained in decreasing direction of field. In this way, a paramagnetic type behavior is shown by both the samples. The magnetic moment of $\mathrm{TaB}_{2.6}$ sample is more than the $\mathrm{TaB}_{2.4}$ sample at a particular field value, which might be due to some magnetic impurity in the boron powder.

Now the point to be discussed is that if increase in $c$ parameter induces superconductivity in $\mathrm{NbB}_{2+x}$, why it does not happen in $\mathrm{TaB}_{2+x}$ case? Actually, if we see the values of $c$ parameter in $\mathrm{NbB}_{2+x}$ case, it has increased from $3.264 \AA$ for pure $\mathrm{NbB}_{2}$ to $3.320 \AA$ for $\mathrm{NbB}_{2.4}$ and saturates thereafter. For $\mathrm{TaB}_{2}, c$ parameter is $3.238 \AA$, which is less than that of pure $\mathrm{NbB}_{2}$. With boron excess, $c$ parameter increases in $\mathrm{TaB}_{2+x}$ case also but slightly, i.e., only up to $3.278 \AA$ for $\mathrm{TaB}_{2.2}$. After that, no increase in $c$ parameter is noticed, which implies excess boron cannot be accommodated in the $\mathrm{TaB}_{2}$ lattice after this limit. Excess boron actually creates metal vacancies in the system as discussed in many theoretical studies. ${ }^{17,18}$ So, we come to the conclusion that although $c$ parameter increases in $\mathrm{TaB}_{2+x}$ case, but it is not sufficient to create enough metal vacancies to introduce superconductivity in this system.

Figure 4 shows the variation in thermoelectric power (TEP) of $\mathrm{MgB}_{2}, \mathrm{AlB}_{2}, \mathrm{NbB}_{2}$ samples with temperature. The $\mathrm{Al}^{+3} / \mathrm{Nb}^{+5}$ substitution at $\mathrm{Mg}^{2+}$ provides extra electrons and hence filling of the hole type sigma band and resulting electron type conductivity while $\mathrm{MgB}_{2}$ is a hole type conductor. As mentioned before, the nonsuperconducting behavior of

TABLE I. Lattice parameters and $c / a$ values for $\mathrm{NbB}_{2+x}$ and $\mathrm{TaB}_{2+x}$ samples with $x=0.0,0.2,0.4,0.6$, and 0.8 .

\begin{tabular}{cllllll}
\hline \hline \multicolumn{3}{c}{$\mathrm{TaB}_{2+x}$} \\
\cline { 2 - 7 }$x$ & \multicolumn{1}{c}{$a(\AA)$} & $c(\AA)$ & $c / a$ & $a(\AA)$ & $\mathrm{NbB}_{2+x}$ \\
\hline \multirow{2}{*}{0.0} & $3.0899(1)$ & $3.2378(2)$ & 1.048 & $3.1103(1)$ & $3.2640(2)$ & 1.049 \\
0.2 & $3.0739(1)$ & $3.2776(2)$ & 1.066 & $3.1013(1)$ & $3.3051(2)$ & 1.066 \\
0.4 & $3.0732(1)$ & $3.2762(2)$ & 1.066 & $3.1041(2)$ & $3.3202(1)$ & 1.069 \\
0.6 & $3.0741(1)$ & $3.2775(1)$ & 1.066 & $3.1018(1)$ & $3.3195(1)$ & 1.070 \\
0.8 & $3.0746(1)$ & $3.2771(2)$ & 1.066 & $3.1040(2)$ & $3.3172(2)$ & 1.069 \\
\hline \hline
\end{tabular}




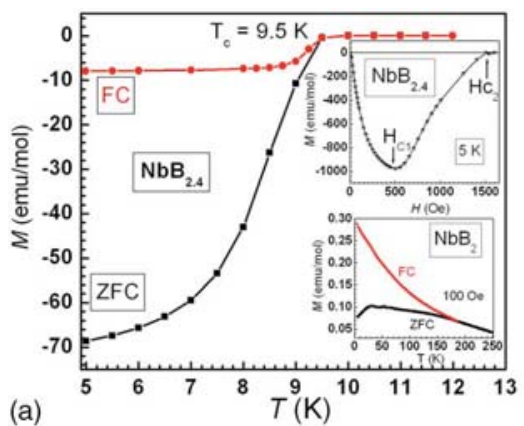

(a)

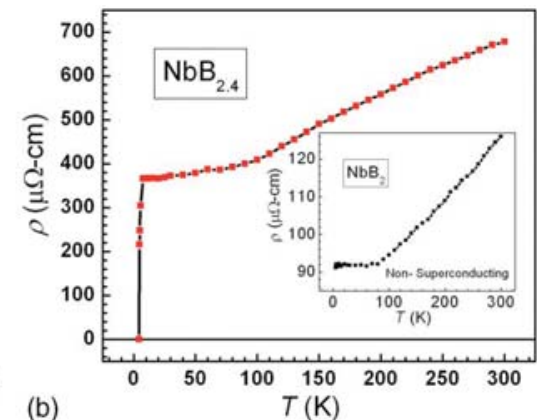

FIG. 2. (Color online) (a) The magnetization vs temperature $(M-T)$ plot for superconducting $\mathrm{NbB}_{2.4}$. The lower inset shows the same for $\mathrm{NbB}_{2}$ while the upper inset shows the $M-H$ plot for $\mathrm{NbB}_{24}$ sample. (b) Variation of resistivity with temperature for $\mathrm{NbB}_{2.4}$ sample. The inset shows the same for $\mathrm{NbB}_{2}$.

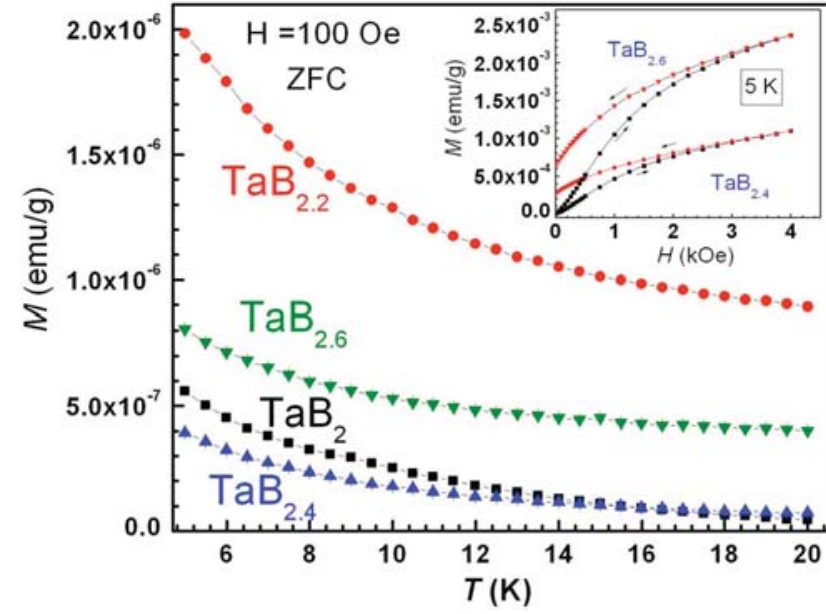

FIG. 3. (Color online) The $M-T$ plot for $\mathrm{TaB}_{2+x}$ sample with $x=0.0,0.2,0.4$, and 0.6. The inset shows the $M-H$ plot for $\mathrm{TaB}_{2.4}$ and $\mathrm{TaB}_{2.6}$ samples.

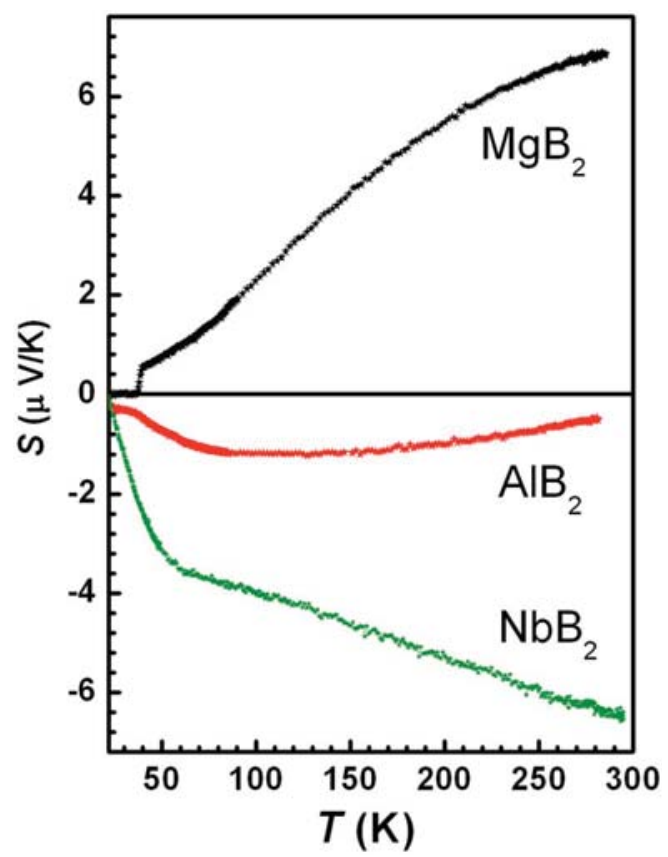

FIG. 4. (Color online) Thermoelectric power vs temperature plots for $\mathrm{MgB}_{2}$, $\mathrm{AlB}_{2}$, and $\mathrm{NbB}_{2}$ samples.
$\mathrm{NbB}_{2}$ and $\mathrm{AlB}_{2}$ is seemingly due to two facts, i.e., changed carrier density and the $c$ parameters. The detailed analysis of TEP data on the basis of two-band model is done earlier for $\mathrm{MgB}_{2}$ and $\mathrm{AlB}_{2} \cdot{ }^{13}$ It is discussed theoretically that the presence of vacancies in the Niobium sublattice of $\mathrm{NbB}_{2}$ brings about considerable changes in the density of states in the near Fermi region and hence affects the superconductivity. ${ }^{19}$

In summary, the $c$ is stretched for nonstoichiometric $\mathrm{NbB}_{2+x}$ and $\mathrm{TaB}_{2+x}$ samples. Excess boron creates metal vacancy in the lattice and induces superconductivity in niobium boride case, but the increase in $c$ parameter is not sufficient in $\mathrm{TaB}_{2}$ case and hence the superconductivity is not achieved. The thermoelectric power measurement shows the different types of carriers in different borides.

${ }^{1}$ J. Nagamatsu, N. Nakagawa, T. Muranaka, Y. Zenitani, and J. Akimitsu, Nature (London) 410, 63 (2001).

${ }^{2}$ V. A. Gasprov, N. S. Sidorov, I. I. Zever'kova, and M. P. Kulakov, JETP Lett. 73, 601 (2001).

${ }^{3}$ L. Leyarovska and E. Leyarovski, J. Less-Common Met. 67, 249 (1979). ${ }^{4}$ H. Rosner, W. E. Pickett, S. L. Drechsler, A. Handstein, G. Behr, G. Fuchs, K. Nankov, K. H. Muller, and H. Eshrig, Phys. Rev. B 64, 144516 (2001).

${ }^{5}$ A. Yamamoto, C. Takao, T. Matsui, M. Izumi, and S. Tajima, Physica C 383, 197 (2002).

${ }^{6}$ D. Kaczorowski, A. J. Zaleski, O. J. Zogal, J. Klamut, e-print arXiv:condmat 0103571 (unpublished).

${ }^{7}$ L. Cooper et al., Proc. Natl. Acad. Sci. U.S.A. 67, 313 (1970).

${ }^{8}$ H. Kotegawa, K. Ishida, Y. Kitaoka, T. Muranaka, H. Takagiwa, and J. Akimitsu, Physica C 378-381, 25 (2002).

${ }^{9}$ J. E. Schirber, D. L. Overmeyer, B. Morosin, E. L. Venturini, R. Baughman, D. Emin, H. Klesnar, and T. Aselage, Phys. Rev. B 45, 10787 (1992).

${ }^{10}$ X. Wan, J. Dong, H. Weng, and D. Y. Xing, Phys. Rev. B 65, 012502 (2001).

${ }^{11}$ V. P. S. Awana, A. Vajpayee, M. Mudgel, V. Ganesan, A. M. Awasthi, G. L. Bhalla, and H. Kishan, Eur. Phys. J. B 62, 281 (2008).

${ }^{12}$ M. Mudgel, V. P. S. Awana, G. L. Bhalla, and H. Kishan, Solid State Commun. 147, 439 (2008)

${ }^{13}$ M. Mudgel, V. P. S. Awana, R. Lal, H. Kishan, L. S. Sharth Chandra, V. Ganesan, A. V. Narlikar, and G. L. Bhalla, J. Phys.: Condens. Matter 20, 095205 (2008).

${ }^{14}$ I. Loa, K. Kunc, K. Syaseen, and P. Bouvier, Phys. Rev. B 66, 134101 (2002).

${ }^{15}$ R. Escamilla, O. Lovera, T. Akachi, A. Duran, R. Falconi, F. Morales, and R. Escudero, J. Phys.: Condens. Matter 16, 5979 (2004).

${ }^{16}$ H. Itoh, Y. Satoh, S. Kodama, and S. Naka, J. Ceram. Soc. Jpn. 98, 264 (1990).

${ }^{17}$ L. E. Muzzy, M. Avdeev, G. Lawes, M. K. Hass, H. W. Zandbergan, A. P. Ramirez, J. D. Jorgensen, and R. J. Cava, Physica C 382, 153 (2002).

${ }^{18} \mathrm{H}$. Klesnar, T. L. Aselage, B. Morosin, and G. H. Kwei, J. Alloys Compd. 241, 180 (1996)

${ }^{19}$ I. R. Shein, N. I. Medvedeva, and A. L. Ivanovskii, Phys. Solid State 45, 1617 (2003). 